\title{
Below-gap excitation of semiconducting single-wall carbon nanotubes
}

\author{
G. Soavi, ${ }^{a, b}$ A. Grupp, ${ }^{a}$ A. Budweg, ${ }^{a}$ F. Scotognella, ${ }^{b, c, d}$ T. Hefner, ${ }^{e}{ }^{\text {T. Hertel, }}{ }^{e}$ \\ G. Lanzani, ${ }^{d}$ A. Leitenstorfer, ${ }^{a}$ G. Cerullob,c and D. Brida*a
}

\begin{abstract}
We investigate the optoelectronic properties of the semiconducting $(6,5)$ species of single-walled carbon nanotubes by measuring ultrafast transient transmission changes with 20 fs time resolution. We demonstrate that photons with energy below the lowest exciton resonance efficiently lead to linear excitation of electronic states. This finding challenges the established picture of a vanishing optical absorption below the fundamental excitonic resonance. Our result points towards below-gap electronic states as an intrinsic property of semiconducting nanotubes.
\end{abstract}

\section{Introduction}

After more than two decades of intense research since their discovery, ${ }^{1,2}$ carbon nanotubes still represent one of the most attractive systems for the study of physical phenomena in one dimension and a promising platform for advanced technology. ${ }^{3,4}$ Single-wall carbon nanotubes (SWNTs) can be described as hollow cylinders obtained by rolling up a graphene sheet. A straightforward and rather precise approach to derive their electronic properties consists in applying the zonefolding scheme (i.e. circumferential periodic boundary conditions) to the electronic bands of graphene obtained by tightbinding (TB) calculations. ${ }^{5}$ These boundary conditions are related to the folding geometry of the cylinder which is unambiguously defined by the chiral vector $\boldsymbol{C}_{\boldsymbol{h}}=\boldsymbol{n} \boldsymbol{a}_{\mathbf{1}}+\boldsymbol{m} \boldsymbol{a}_{\mathbf{2}}$. Here, $\boldsymbol{a}_{1}$ and $\boldsymbol{a}_{2}$ are the unit vectors of the graphene lattice. ${ }^{6}$ From the pair of integers $(\boldsymbol{n}, \boldsymbol{m})$ it is also possible to predict whether a SWNT will be metallic or semiconducting, ${ }^{5,6}$ with an energy gap scaling as the inverse of the tube diameter in the latter case. $^{7,8}$ This single-particle model has to be refined by considering many-body effects that lead to the formation of excitons, ${ }^{9-12}$ bi-excitons ${ }^{13,14}$ and trions. ${ }^{15-17}$ In addition, a more detailed TB description of the electronic structure must take curvature into account, in particular at SWNT diameters

${ }^{a}$ Department of Physics and Center for Applied Photonics, University of Konstanz, D 78457 Konstanz, Germany. E mail: gs544@cam.ac.uk, daniele.brida@uni konstanz.de

${ }^{b}$ Dipartimento di Fisica, Politecnico di Milano, Piazza L. da Vinci 32, 20133 Milano, Italy

${ }^{c}$ IFN CNR, Piazza L. da Vinci, 32, 20133 Milano, Italy

${ }^{d}$ Center for Nano Science and Technology@PoliMi, Istituto Italiano di Tecnologia, Via Giovanni Pascoli, 70/3, 20133 Milano, Italy

${ }^{e}$ Inst. for Physical and Theoretical Chemistry Dept. of Chemistry and Pharmacy, University of Würzburg, Würzburg 97074, Germany below about $1 \mathrm{~nm} \cdot{ }^{5,18-21}$ This effect is responsible for both the formation of a small band gap in metallic $\operatorname{SWNTs}^{22}$ and a drastic reduction of the band gap in small-diameter semiconducting SWNTs. ${ }^{23}$ Also defects, disorder and doping, including ambient contamination from water and oxygen during the synthesis, may strongly affect the electronic and transport properties of SWNTs. ${ }^{24-26}$ Such extrinsic effects lead to phenomena like e.g. a plasma response in the THz frequency range,$^{27}$ local variations of the Fermi level ${ }^{28}$ and the appearance of an Urbach tail in the effective density of states. ${ }^{29}$ Finally, the intrinsic electronic properties of SWNTs may be modified by the presence of bundles which lead to lowering of the symmetry. ${ }^{5}$ However, below-gap excitation in semiconducting SWNTs has been rarely investigated experimentally. In a previous study on HiPco (high-pressure carbon monoxide) SWNTs, ${ }^{30}$ the obtained signal was assigned to thermal effects or to a non-resonant bleaching from large-diameter SWNTs. In another study, Song et al..$^{31}$ observed a long-lived bleaching of the $S_{11}$ transition in a $(6,5)$-enriched sample which they attributed to weak excitation of real carriers through multiphoton absorption. On the other hand, absorption spectroscopy on individual and relatively large-diameter SWNTs hints to the presence of a "non-resonant" background with a cross-section comparable to the ideal graphene optical absorbance, regardless of the polarization (i.e. parallel or orthogonal to the tube axis) of the incident light. ${ }^{32}$ Similarly, recent photoluminescence experiments showed a graphene-like wavelength-independent absorption at higher energy with respect to the $\mathrm{S}_{11}$ excitonic transition in small-diameter semiconducting SWNTs, ${ }^{33}$ including the $(6,5)$ chirality. In this work we apply ultrafast pump-probe spectroscopy to investigate the nature of excitations at low photon energy (i.e. below the fundamental gap) in small-diameter semiconducting SWNTs. We unambiguously show that optical excitation can occur below 
the excitonic gap without exploiting non-linear processes such as two-photon absorption. This result illustrates photo-physical phenomena in small-diameter semiconducting SWNTs beyond the conventional exciton physics. We suggest that significant theoretical and experimental efforts are still necessary to fully capture their energetic landscape.

\section{Experimental}

\subsection{Sample preparation}

The sample used for the experiments is highly enriched to contain a fraction of more than $80 \%$ of the $(6,5)$ semiconducting species of SWNTs embedded in a gelatine film. It was prepared from 30 microliters of a density gradient ultracentrifugation-enriched (DGU) SWNT suspension in a sodium cholate (SC)/sodium dodecyl sulfate (SDS) mixture. ${ }^{34}$ Iodixanol as well as SDS residues from the DGU process were removed by dilution with SC solution and filtration with a benchtop centrifuge. The resulting suspension was then mixed with 20 microliters of $15 \mathrm{wt} \%$ gelatine solution and finally drop-cast onto a thin glass substrate. The $(6,5)$ chirality, characterized by a $0.75 \mathrm{~nm}$ diameter and a band gap of $1.26 \mathrm{eV}$, has been widely used to study the fundamental physical properties of semiconducting SWNTs. ${ }^{17,28,35-38}$ This fact is mostly due to convenient spectroscopic access to the lowest subband exciton, abundance and ease of isolation if compared with other semiconducting species. ${ }^{34,39}$

\subsection{Pump-probe experiments}

Transient absorption spectroscopy was performed with a home-built system based on a $50 \mathrm{kHz}$ Yb:KGW regenerative amplifier system (PHAROS, Light Conversion) that drives a tuneable IR optical parametric amplifier (OPA) delivering the pump pulses. As a probe we employ a white-light continuum (WLC) generated in a $4 \mathrm{~mm}$ sapphire plate. The pulses are compressed by means of a prisms pair (IR OPA) and by chirped mirrors (WLC), ensuring an overall temporal resolution of approximately $20 \mathrm{fs}$. The spectra of the three different pump pulses, obtained by tuning the IR OPA, are reported in Fig. 1 together with the absorption spectrum of the $(6,5)$ SWNT sample in the energy region of the first two excitonic transitions, $S_{11}$ and $S_{22}$. The photon energies of the pump pulses (from $1 \mathrm{eV}$ to $0.82 \mathrm{eV}$ ) are tuned to be well below the first $S_{11}$ excitonic transition of the $(6,5) \mathrm{SWNT}$ at $1.26 \mathrm{eV}$. The probe pulse is spectrally resolved by a monochromator and lock-in detected by modulating the pump beam with a Pockels cell at $25 \mathrm{kHz}$ frequency.

\subsection{Transient spectra and coherent phonons}

Fig. 2 shows the measured differential transmission $(\Delta T / T)$ signal in the energy region of the $\mathrm{S}_{22}$ excitonic transition for excitation by photons with $0.9 \mathrm{eV}$ energy. The results are similar for the three pump photon energies discussed in Fig. 1. Both the overall spectral shape and its ultrafast evolution (Fig. 2) strongly resemble the typical response of a $(6,5)$

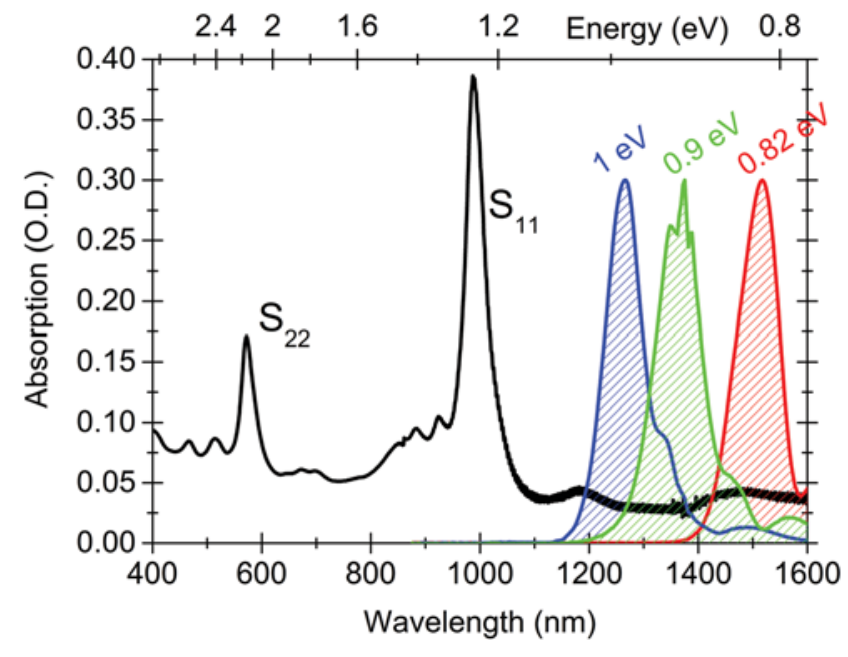

Fig. 1 Absorption spectrum of the $(6,5)$ SWNT and the three pump pulses used in the pump probe experiments. The first two excitons $S_{11}$ and $S_{22}$ reside at wavelengths of approximately $1 \mu \mathrm{m}$ and $570 \mathrm{~nm}$, respectively.

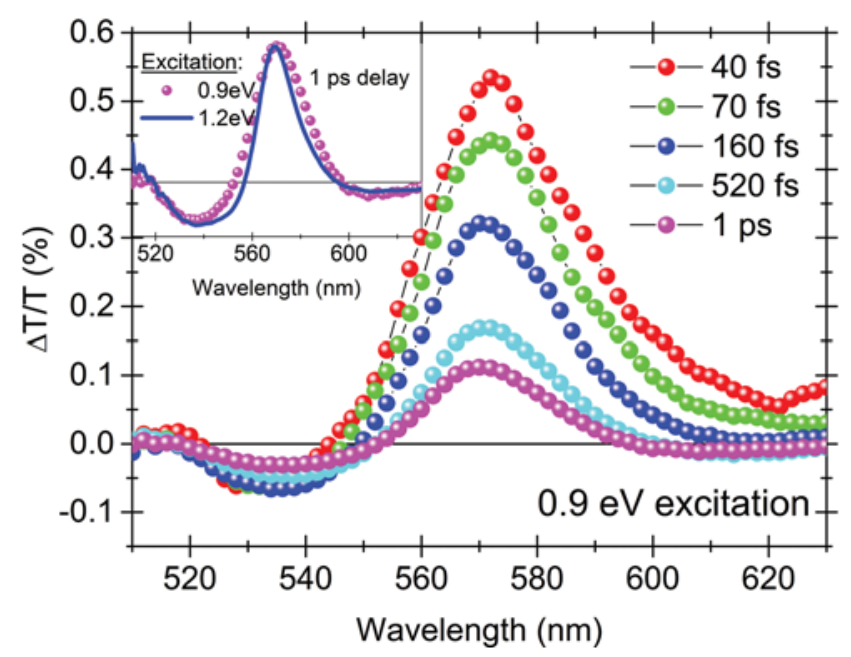

Fig. 2 Differential transmission signal $\Delta T / T$ at different pump probe delays in the case of excitation at a photon energy of $0.9 \mathrm{eV}$. (inset) Comparison between normalized $\Delta T / T$ signal for $0.9 \mathrm{eV}$ (circles) and $1.2 \mathrm{eV}$ excitation (blue line), resonant with the $S_{11}$ exciton, acquired at 1 ps pump probe delay.

SWNT resonantly excited at the $\mathrm{S}_{11}$ transition, ${ }^{40-42}$ despite the pump pulses in this study have much lower photon energy.

Fig. 3a displays the $\Delta T / T$ dynamics monitored by probe pulses at $570 \mathrm{~nm}$ and $580 \mathrm{~nm}$ wavelength when the sample is excited with photons of $1 \mathrm{eV}$ energy. The inset highlights that the response of the sample is instantaneous within our temporal resolution of $20 \mathrm{fs}$. To confirm that the sample is excited via a linear process, and thus to exclude a two-photon excitation, we performed pump-probe measurements as a function of the excitation fluence at the peak of the $S_{22}$ bleaching $(570 \mathrm{~nm})$. Fig. $3 \mathrm{~b}$ demonstrates that the signal scales linearly 

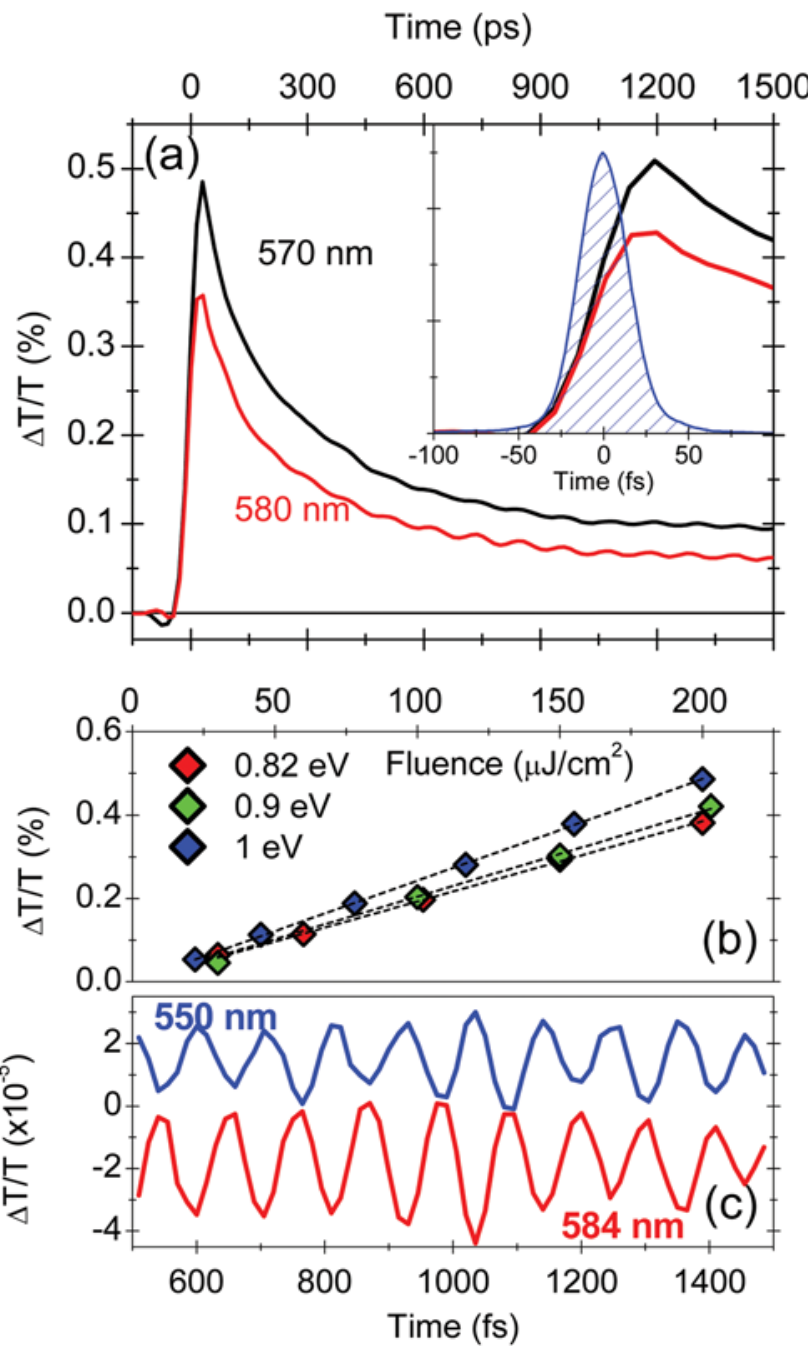

Fig. 3 (a) $\Delta T / T$ dynamics at $570 \mathrm{~nm}$ and $580 \mathrm{~nm}$ probe wavelengths. (inset) Zoom in of the dynamics between $100 \mathrm{fs}$ and $100 \mathrm{fs}$ together with the auto correlation of the pump pulse demonstrating a temporal resolution of approximately $20 \mathrm{fs}$. (b) Maximum amplitude of the pump probe signal for the $570 \mathrm{~nm}$ probe wavelength as a function of the pump fluence and linear fits. (c) Coherent oscillations (assigned to the RBMs) detected at $584 \mathrm{~nm}$ (blue curve) and $550 \mathrm{~nm}$ (red curve) for $1 \mathrm{eV}$ pump photon energy.

with the excitation over more than one order of magnitude, while Fig. 4a shows a very weak dependence of the decay constant on the pump fluence. The $\Delta T / T$ dynamics at $570 \mathrm{~nm}$ probe wavelength is depicted in Fig. $4 \mathrm{~b}$ as a function of the pump-photon energy. The amplitude of the transient signal is virtually independent of the photon energy of the pump pulse (see Fig. 3b). However, at higher pump photon energy the relaxation becomes slightly faster, as it is typical for the thermalization processes that follow excitation within a continuum of states. ${ }^{43}$ In addition, we clearly observe that the dynamics of $\Delta T / T$ is always modulated by periodic oscillations of the amplitude. The frequency of these oscillations of $310 \mathrm{~cm}^{-1}$, as well as their modulation depth and phase profile, allows us to ascribe them to an impulsively excited radial breathing mode

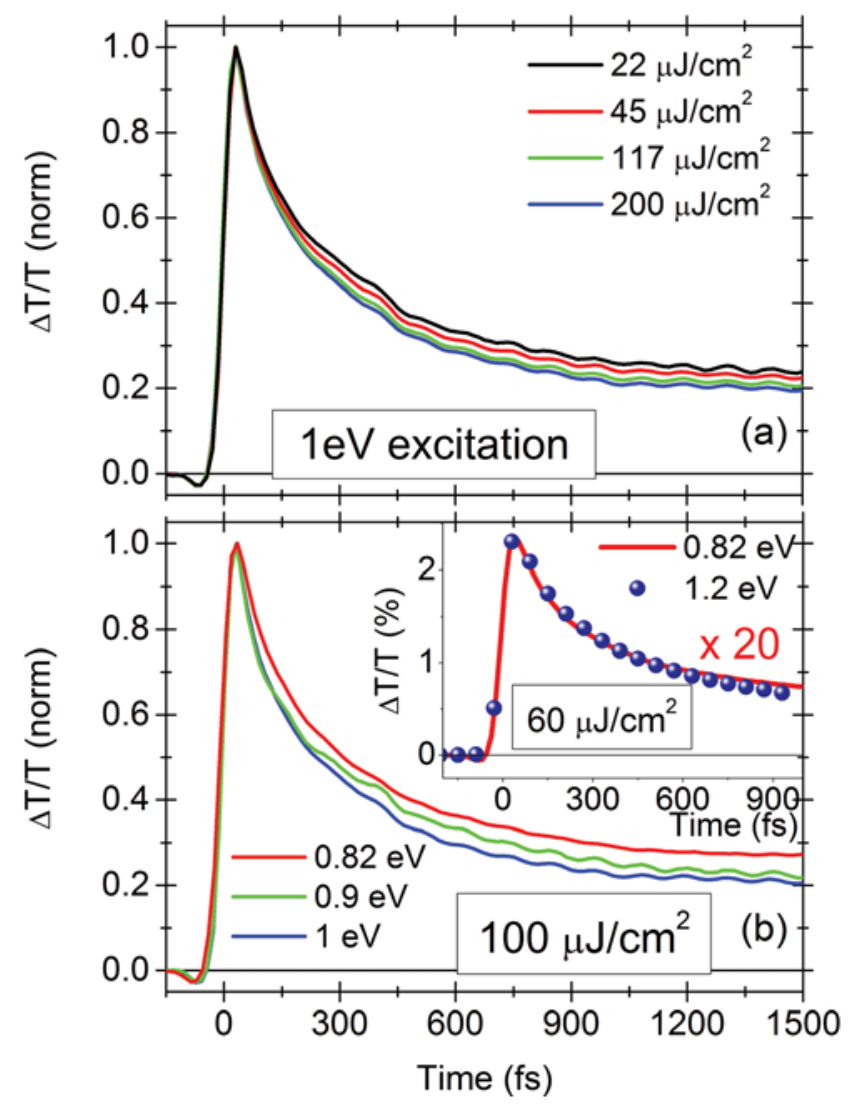

Fig. 4 (a) Fluence dependent dynamics at $570 \mathrm{~nm}$ probe wavelength. (b) Dynamics at $570 \mathrm{~nm}$ probe wavelength and $100 \mu \mathrm{J} \mathrm{cm}{ }^{2}$ pump fluence for the three different pump spectra used in the experiments. (inset) Comparison between dynamics after excitation off resonance at $0.82 \mathrm{eV}$ (solid red line) and resonant pumping of the $S_{11}$ exciton at $1.2 \mathrm{eV}$ (circles) at a pump fluence of $60 \mu \mathrm{J} \mathrm{cm}{ }^{2}$. The $\Delta T / T$ signal for $0.82 \mathrm{eV}$ excitation is multiplied by a factor of 20 .

(RBM) of the $(6,5)$ SWNT. In fact, the Fourier amplitude of these coherent phonon oscillations vanishes at the peak of the bleaching (see the dynamics at $570 \mathrm{~nm}$ probe wavelength in Fig. 3a) and it exhibits maxima in its slopes (see the dynamics at $550 \mathrm{~nm}$ and $584 \mathrm{~nm}$ probe wavelengths in Fig. 3c, respectively), with a clear jump in the phase by $\pi$ between lower and higher probe energies (Fig. 3c). ${ }^{44,45}$ Vibrational coherence can only be established by an impulsive excitation occurring on a time scale $\Delta t \ll \tau$ where $\tau$ is the vibrational period of the specific mode. In our case, $\tau$ amounts to $110 \mathrm{fs}$. Off-resonance impulsive stimulated Raman scattering is unlikely in a highly diluted sample where the matrix signal would vastly dominate. Also, it would not display the measured phase profile, with a phase jump by $\pi$ around the excitonic resonance. In combination with the observation of a bleaching signal, our findings demonstrate that below-gap excitation creates a real population of SWNT electronic states by a dissipative process. This scenario is in stark contrast with coherent nonlinear interactions involving transient shifts of energy levels in the presence of a non-resonant light field as e.g. the optical Stark 
effect. ${ }^{46}$ Such signals would last only for the temporal overlap of pump and probe pulses and would not cause a long-lived bleaching. Note that even the linear absorption spectrum of the sample, shown in Fig. 1, displays a nearly featureless lowenergy tail. While from linear absorption it is hard to discriminate the contributions of the SWNTs from that of the gelatine, scattering and impurities, our experiments clearly demonstrate that at least part of this absorption directly results from the intrinsic properties of the $(6,5)$ SWNTs.

\section{Results and discussion}

Based on this experimental evidence, we now discuss the possible origin of the $\Delta T / T$ signal following low-energy optical excitation. (i) Since the pump photon energies are well below the $\mathrm{S}_{11}$ main peak transition energy, direct exciton photogeneration can be excluded. (ii) Another possibility is that excitons might be created via two-photon absorption with subsequent onset of exciton-exciton annihilation, resulting in linear fluence dependence. However, this scenario can be ruled out by considering that the photon energies employed in our experiments are not resonant with $\mathrm{S}_{11}$ via a two-photon transition. In addition, exciton-exciton annihilation can only occur at high densities of photoexcited states, which are extremely unlikely with our moderate pump fluences and assuming a two-photon absorption mechanism (Fig. 3b). Finally, the amplitude of the $\mathrm{S}_{22} \Delta T / T$ signal and the decay dynamics of the exciton bleaching should display non-linear dependence on the pump fluence, ${ }^{38,47,48}$ which is not the case in our experiments as reported in Fig. 4a. (iii) Thermal effects, for instance due to heating of the matrix, would not be instantaneous in the tube response and should persist on a timescale much longer than the ps decay we observe. In fact, heat transfer from the environment to the $(6,5)$ SWNT would imply a rise-time of the signal in the picosecond range as typical for lattice heating dynamics. ${ }^{49}$ (iv) Absorption of low-energy photons with the corresponding annihilation of phonons is ruled out by the large tuning range of the pump pulses used in the experiments (which do not match with any of the $(6,5)$ SWNT phonons) and the negligible population of high-energy phonons available at room temperature. To further exclude such phonon-assisted exciton generation mechanism, we have compared photoluminescence (PL) experiments following resonant $\mathrm{S}_{22}(2.17 \mathrm{eV})$ and below-gap $(0.9 \mathrm{eV})$ excitation. The PL signal was collected at the peak of the $S_{11}$ exciton $(1.2 \mathrm{eV})$ in both cases. In these experiments we observed a clear and sharp PL signal in the case of $S_{22}$ excitation while we did not observe any PL from below-gap excitation. (v) Our observation also rules out excitation and energy transfer from residual metallic or semiconducting tubes of other chiralities present in the sample, since energy transfer from an unknown absorber to the $(6,5)$ SWNT would be an unlikely up-hill event. (vi) Finally, even in the case of unintentional p-doping of SWNTs, for instance due to interaction with the oxygen-water redox couple, absorption of light with polarization perpendicu-

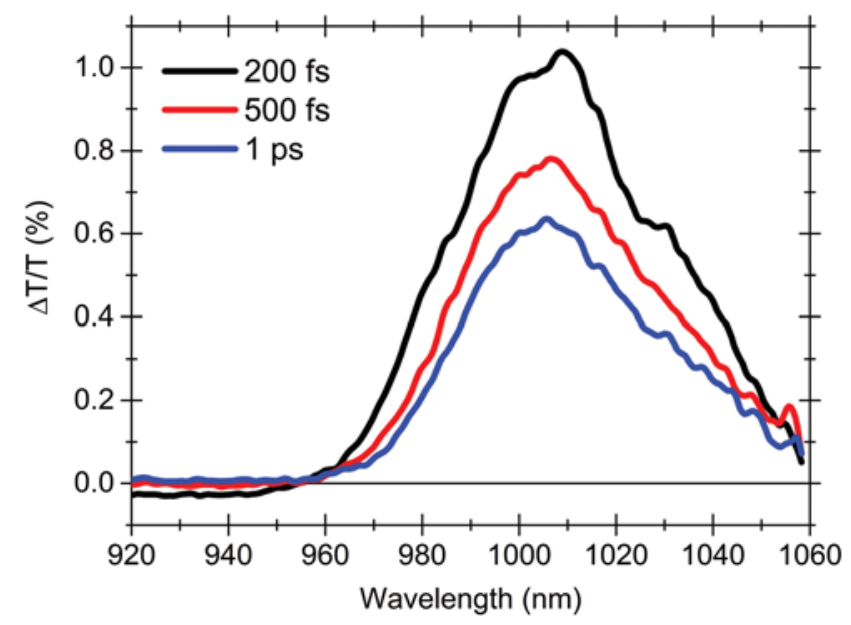

Fig. $5 \Delta T / T$ spectra at different pump probe delays in the energy region of the first $S_{11}$ excitonic subband in the case of below gap $(0.9 \mathrm{eV})$ excitation and $\approx 200 \mu \mathrm{J} \mathrm{cm}^{2}$ fluence.

lar to the tube axis might induce transitions between the $S_{22}$ valence band and vacancies around the top of the $S_{11}$ excitonic subband. We addressed this issue by probing the first excitonic subband in the case of below-gap $(0.9 \mathrm{eV})$ excitation. The results (Fig. 5) show a clear bleaching signal from the $S_{11}$ exciton, which again strongly resemble the case of resonant or above-gap excitation. ${ }^{37}$ This allows us to exclude the aforementioned scenario, for which an increased absorption would be expected.

These experimental results allow us to assign the absorption of low-energy photons directly to linear excitation of the $(6,5)$ SWNTs. The evidence that the amplitude of the signal does not depend on the pump photon energy (Fig. 3b) further indicates that this excitation is associated with a distributed joint density of states directly related to the $(6,5)$ SWNT. Similarly, since free charges are also strongly coupled to the RBMs of individual SWNTs, ${ }^{50}$ the perturbation of the $(6,5)$ electronic distribution can explain the presence of the coherent oscillations via electron-phonon coupling, without invoking excitonic effects. By comparing the $\Delta T / T$ signal amplitude in the case of resonant $S_{11}$ (inset in Fig. 2 and inset in Fig. 4b) and resonant $\mathrm{S}_{22}$ excitation from ref. 35 with that of below-gap excitation, we are able to estimate a below-gap optical density (in the energy region between 0.8 and $1 \mathrm{eV}$ investigated here) of approximately $0.07-0.012$. Our experiments also indicate that below-gap excitation produces the same transient spectra (inset Fig. 2 and 5) and dynamics (inset Fig. 4b) when compared to resonant excitation of the $\mathrm{S}_{11}$ transition. This result agrees with our previous studies, ${ }^{42,51}$ where we show that the pump-probe signal is strongly affected by photo-generated free electron-hole pairs. In fact, the optical properties of SWNTs depend on the presence of optically excited or injected charges due to band-gap renormalization. ${ }^{42,51-53}$ The influence of defects or doping ${ }^{24-29}$ would result in shallow or deep traps: ${ }^{26}$ the low energy of the pump photons and their broad tuning 
(from $1 \mathrm{eV}$ to $0.8 \mathrm{eV}$ ) would require an extremely high and uniform level of doping which is unlikely with our sample quality. Nevertheless, we cannot firmly exclude the presence of such states and hopefully this work will trigger theoretical investigations in this direction. On the other hand, the nature of this below-gap continuum of electronic states may be intrinsic to the $(6,5)$ SWNT and explained by the effect of finite curvature. ${ }^{18}$ These findings might also explain some controversial observations such as the low photo-luminescence quantum yield in small diameter SWNTs ${ }^{54}$ or linear charge photogeneration upon excitation in the energy region of the $S_{11}$ exciton. ${ }^{55,56}$

\section{Conclusions}

In conclusion, our results unambiguously show that at frequencies below the well-known $S_{11}$ singlet exciton resonance of SWNTs there exist transitions with non-negligible oscillator strength. Such transitions generate real population, causing state filling, bleaching of higher energy optical transitions and coherent phonons. These observations are consistent with a graphene-like electronic density of states that is associated to a continuum in the spectral response. This fact may be described by models including defects, doping or curvature effects and subsequent hybridization of orbitals at the tube surface. These results are thus challenging the commonly accepted picture for the electronic structure of semiconducting SWNTs, warranting more refined theoretical descriptions that take into account e.g. deviations from linear geometry due to bending.

\section{Acknowledgements}

D. B. acknowledges the support of the Emmy Noether Program of the Deutsche Forschungsgemeinschaft (DFG), of Zukunftskolleg and of EC through the Marie Curie CIG project "UltraQuEsT" no. 334463. C. G. and G. S. acknowledge support by the EC under Graphene Flagship (contract no. CNECT-ICT-604391). F. S., G. L. and T. H. acknowledge the ITN project 316633 "POCAONTAS". A. L. acknowledges the European Research Council Advanced Grant "UltraPhase" (ERC-2011-AdG no. 290876).

\section{Notes and references}

1 S. Iijima, Nature, 1991, 354, 56.

2 S. Iijima and T. Ichihashi, Nature, 1993, 363, 603.

3 R. H. Baughman, A. A. Zakhidov and W. A. de Heer, Science, 2002, 297, 787.

4 M. F. De Volder, S. H. Tawfick, R. H. Baughman and A. J. Hart, Science, 2013, 339, 535.

5 J.-C. Charlier, X. Blase and S. Roche, Rev. Mod. Phys., 2007, 79, 677.

6 R. Saito, G. Dresselhaus and M. S. Dresselhaus, Physical Properties of Carbon Nanotubes, Imperial college press, London, 1998.
7 C. White and J. Mintmire, Nature, 1998, 394, 29.

8 H. Kataura, Y. Kumazawa, Y. Maniwa, I. Umezu, S. Suzuki, Y. Ohtsuka and Y. Achiba, Synth. Met., 1999, 103, 2555.

9 T. Ando, J. Phys. Soc. Jpn., 1997, 66, 1066.

10 C. D. Spataru, S. Ismail-Beigi, L. X. Benedict and S. G. Louie, Phys. Rev. Lett., 2004, 92, 077402.

11 F. Wang, G. Dukovic, L. E. Brus and T. F. Heinz, Science, 2005, 308, 838.

12 J. Maultzsch, R. Pomraenke, S. Reich, E. Chang, D. Prezzi, A. Ruini, E. Molinari, M. Strano, C. Thomsen and C. Lienau, Phys. Rev. B: Condens. Matter, 2005, 72, 241402.

13 T. G. Pedersen, K. Pedersen, H. D. Cornean and P. Duclos, Nano Lett., 2005, 5, 291.

14 L. Colombier, J. Selles, E. Rousseau, J. Lauret, F. Vialla, C. Voisin and G. Cassabois, Phys. Rev. Lett., 2012, 109, 197402.

15 T. Rønnow, T. Pedersen and H. Cornean, Phys. Rev. B: Condens. Matter, 2010, 81, 205446.

16 K. Watanabe and K. Asano, Phys. Rev. B: Condens. Matter, 2012, 85, 035416.

17 S. M. Santos, B. Yuma, S. Berciaud, J. Shaver, M. Gallart, P. Gilliot, L. Cognet and B. Lounis, Phys. Rev. Lett., 2011, 107, 187401.

18 X. Blase, L. Benedict, E. Shirley and S. Louie, Phys. Rev. Lett., 1994, 72, 1878.

19 A. Kleiner and S. Eggert, Phys. Rev. B: Condens. Matter, 2001, 64, 113402.

20 V. Zólyomi and J. Kürti, Phys. Rev. B: Condens. Matter, 2004, 70, 085403.

21 I. Cabria, J. Mintmire and C. White, Phys. Rev. B: Condens. Matter, 2003, 67, 121406.

22 M. Ouyang, J. L. Huang, C. L. Cheung and C. M. Lieber, Science, 2001, 292, 702.

23 Y. Matsuda, J. Tahir-Kheli and W. Goddard, J. Phys. Chem. Lett., 2010, 1, 2946.

24 D. Kang, N. Park, J. Ko, E. Bae and W. Park, Nanotechnology, 2005, 16, 1048.

25 P. G. Collins, K. Bradley, M. Ishigami and A. Zettl, Science, 2000, 287, 1801.

26 S. Lee, G. Kim, H. Kim, B.-Y. Choi, J. Lee, B. W. Jeong, J. Ihm, Y. Kuk and S.-J. Kang, Phys. Rev. Lett., 2005, 95, 166402.

27 Q. Zhang, E. H. Hároz, Z. Jin, L. Ren, X. Wang, R. S. Arvidson, A. Lüttge and J. Kono, Nano Lett., 2013, 13, 5991.

28 S. Schafer, N. B. Cogan and T. D. Krauss, Nano Lett., 2014, 14, 3138.

29 D. Jones and J. Lee, Nano Lett., 2011, 11, 4176.

30 R. Ellingson, C. Engtrakul, M. Jones, M. Samec, G. Rumbles, A. Nozik and M. Heben, Phys. Rev. B: Condens. Matter, 2005, 71, 115444.

31 D. Song, F. Wang, G. Dukovic, M. Zheng, E. D. Semke, L. E. Brus and T. Heinz, Appl. Phys. A, 2009, 96, 283.

32 J.-C. C. Blancon, M. Paillet, H. N. Tran, X. T. Than, S. A. Guebrou, A. Ayari, A. S. Miguel, N.-M. M. Phan, A.-A. 
A. Zahab, J.-L. L. Sauvajol, N. D. Fatti and F. Vallée, Nat. Commun., 2013, 4, 2542.

33 F. Vialla, E. Malic, B. Langlois, Y. Chassagneux, C. Diederichs, E. Deleporte, P. Roussignol, J.-S. Lauret and C. Voisin, Phys. Rev. B: Condens. Matter, 2014, 90, 155401.

34 J. Crochet, M. Clemens and T. Hertel, J. Am. Chem. Soc., 2007, 129, 8058.

35 J. R. Schneck, A. G. Walsh, A. A. Green, M. C. Hersam, L. D. Ziegler and A. K. Swan, J. Phys. Chem. A, 2011, 115, 3917.

36 J. Blackburn, J. Holt, V. Irurzun, D. Resasco and G. Rumbles, Nano Lett., 2012, 12, 1398.

37 Z. Zhu, J. Crochet, M. S. Arnold, M. C. Hersam, H. Ulbricht, D. Resasco and T. Hertel, J. Phys. Chem. C, 2007, 111, 3831.

38 L. Lüer, S. Hoseinkhani, D. Polli, J. Crochet, T. Hertel and G. Lanzani, Nat. Phys., 2009, 5, 54.

39 S. Bachilo, L. Balzano, J. Herrera, F. Pompeo, D. Resasco and R. Weisman, J. Am. Chem. Soc., 2003, 125, 1186.

40 J. Park, P. Deria and M. J. Therien, J. Am. Chem. Soc., 2011, 133, 17156

41 J. Crochet, S. Hoseinkhani, L. Lüer, T. Hertel, S. Doorn and G. Lanzani, Phys. Rev. Lett., 2011, 107, 257402.

42 G. Soavi, F. Scotognella, D. Brida, T. Hefner, F. Späth, M. Antognazza, T. Hertel, G. Lanzani and G. Cerullo, J. Phys. Chem. C, 2013, 117, 10849.

43 G. Della Valle, M. Conforti, S. Longhi, G. Cerullo and D. Brida, Phys. Rev. B: Condens. Matter, 2012, 86, 155139.
44 Y.-S. S. Lim, K.-J. J. Yee, J.-H. H. Kim, E. H. Hároz, J. Shaver, J. Kono, S. K. Doorn, R. H. Hauge and R. E. Smalley, Nano Lett., 2006, 6, 2696.

45 L. Lüer, C. Gadermaier, J. Crochet, T. Hertel, D. Brida and G. Lanzani, Phys. Rev. Lett., 2009, 102, 127401.

46 T. Unold, K. Mueller, C. Lienau, T. Elsaesser and A. D. Wieck, Phys. Rev. Lett., 2004, 92, 157401.

47 L. Valkunas, Y.-Z. Ma and G. R. Fleming, Phys. Rev. B: Condens. Matter, 2006, 73, 115432.

48 F. Wang, G. Dukovic, E. Knoesel, L. E. Brus and T. F. Heinz, Phys. Rev. B: Condens. Matter, 2004, 70, 241403(R).

49 G. V. Hartland, Chem. Rev., 2011, 111, 3858.

50 B. J. LeRoy, S. G. Lemay, J. Kong and C. Dekker, Nature, 2004, 432, 371.

51 G. Soavi, F. Scotognella, D. Viola, T. Hefner, T. Hertel, G. Cerullo and G. Lanzani, Sci. Rep., 2015, 5, 9681.

52 M. Steiner, M. Freitag, V. Perebeinos, A. Naumov, J. P. Small, A. A. Bol and P. Avouris, Nano Lett., 2009, 9, 3477.

53 W. J. Kennedy and Z. V. Vardeny, Appl. Phys. Lett., 2011, 98, 263110.

54 P. Avouris, M. Freitag and V. Perebeinos, Nat. Photonics, 2008, 2, 341.

55 M. Beard, J. L. Blackburn and M. Heben, Nano Lett., 2008, 8, 4238 .

56 S. A. Jensen, R. Ulbricht, A. Narita, X. Feng, K. Müllen, T. Hertel, D. Turchinovich and M. Bonn, Nano Lett., 2013, 13, 5925 . 